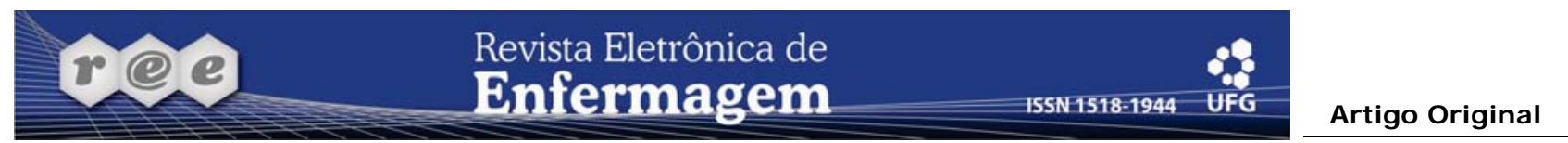

\title{
Qualidade de vida em pacientes submetidos à prostatectomia radical
}

Quality of life in patients submitted to radical prostatectomy

Calidad de vida en pacientes sometidos a prostatectomía radical

Débora Moura Miranda Goulart ${ }^{1}$, Mário Alfredo Silveira Miranzi ${ }^{2}$, Paulo Eduardo Nunes Goulart ${ }^{3}$

${ }^{1}$ Enfermeira, Mestre em Atenção à Saúde. Professora da Universidade de Uberaba. Uberaba, MG. Brasil. E-mail: debysmm@gmail.com.

2 Odontólogo, Doutor em saúde Coletiva. Professor Adjunto da Universidade Federal do Triângulo Mineiro (UFTM). Uberaba, MG. Brasil. E-mail:

mmiranzi@mednet.com.br.

${ }^{3}$ Médico. Uberaba, MG. Brasil. E-mail: nunesgoulart@gmail.com.

\section{RESUMO}

Estudo transversal, com análise descritiva de 81 pacientes submetidos à prostatectomia radical (PR), cujo objetivo foi relacionar a qualidade de vida (QV), segundo o EORTC-QLQ C30, com a faixa etária e tempo pós-operatório. A média etária foi de 65,7 anos. A maioria procurou a urologia, assintomáticos. Referiram ex-tabagismo $(49,4 \%)$ e hipertensão arterial $(53,1 \%)$. A média do PSA pré-operatório foi de $8,4 \mathrm{ng} / \mathrm{ml}$. Prevaleceram estádios de T2c a T3, Gleason $\leq 6 \mathrm{e}$ tempo pós-operatório maior que um ano. Apresentaram disfunção erétil 90,1\% e incontinência urinária 33,3\%. A QV apresentou altos índices nas escalas funcionais e global de saúde e baixos na sintomatologia. Verificou-se melhor QV nos idosos nos itens: função emocional, dificuldade financeira e saúde global e, naqueles com mais de um ano de cirurgia, nos itens: função cognitiva e fadiga. Embora não tenha sido observado grande impacto na QV, houve diferenças entre os grupos etários e o tempo pós-operatório.

Descritores: Qualidade de Vida; Prostatectomia; Disfunção Erétil; Incontinência Urinária.

\section{ABSTRACT}

A cross-sectional study with a descriptive analysis of 81 patients submitted to radical prostatectomy (RP). Our objective was to correlate quality of life (QL) according to the EORTC-QLQ C30 with age group and time after surgery. Mean age was 65.7 years. Most sought the care of urology, asymptomatic. Some referred former smoking (49.9\%) and high blood pressure (53.1\%). Mean preoperative SBP was $8.4 \mathrm{ng} / \mathrm{ml}$. Most participants were in stages T2c to T3, Gleason $\leq 6$ and over a year after surgery. Erectile dysfunction presented in $90.1 \%$, and urinary incontinence in 33.3\%. Functional and overall health scales presented high QL indexes, and symptomatology, with low ones. Older adults presented higher QL regarding emotional functioning, financial difficulties and overall health, as well as those with over a year after surgery, regarding cognitive functioning and fatigue. Although QL was not greatly affected, there were differences between age groups and time after surgery.

Descriptors: Quality of Life; Prostatectomy; Erectile Dysfunction; Urinary Incontinence.

\section{RESUMEN}

Estudio transversal con análisis descriptivo de 81 pacientes sometidos a prostatectomía radical (PR), objetivando relacionar la calidad de vida (QV) según el EORTC-QLQ C30 con la faja etaria y tiempo postoperatorio. La media etaria fue de 65,7 años. La mayoría se atendió en urología, asintomáticos. Se informaron como ex fumadores (49.4\%) e hipertensión arterial $(53,1 \%)$. El PSA preoperatorio promedio fue 8,4 ng/ml. Prevalecieron estadios T2c a T3, Gleason $\leq 6$, tiempo postoperatorio superior al año. Presentaron disfunción eréctil 90,1\% e incontinencia urinaria 33,3\%. La QV expresó índices altos en escalas funcional y global de salud, y bajos en sintomatología. Se verificó mejor QV en ancianos en los ítems: función emocional, dificultad financiera y salud global; y en aquellos con más de un año de cirugía en los ítems: función cognitiva y fatiga. Aunque no se haya observado gran impacto en la QV, existieron diferencias entre grupos etarios y tiempo postoperatorio.

Descriptores: Calidad de Vida; Prostatectomía; Disfunción Eréctil; Incontinencia Urinaria. 


\section{NTRODUÇÃO}

Atualmente, o câncer é uma das principais causas de morte no mundo, sendo considerado um problema de saúde pública em países desenvolvidos e em desenvolvimento. A Organização Mundial da Saúde (OMS) estimou que, no ano 2030, podem-se esperar 27 milhões de casos incidentes de câncer, 17 milhões de mortes por câncer e 75 milhões de pessoas vivas, anualmente, com câncer. A última estimativa mundial apontou o câncer da próstata (CaP) como sendo o segundo tipo de câncer mais frequente em homens, cerca de 1,1 milhão de casos novos no ano de $2012^{(1)}$.

No Brasil, a estimativa para o ano de 2014, válida também para o ano de 2015, reforça a magnitude do problema. São esperados aproximadamente 576 casos novos, sendo 68.800 de CaP, o que corresponde a um risco estimado de 70,42 casos novos a cada 100.000 homens. O CaP continua sendo a segunda neoplasia mais comum entre os homens. O aumento da expectativa de vida, a melhoria e a evolução dos métodos diagnósticos e da qualidade dos sistemas de informação do país podem explicar o aumento das taxas de incidência ao longo dos anos ${ }^{(1)}$.

Apesar dessas estimativas ainda são observados poucos estudos em relação à saúde do homem com câncer, mesmo naqueles de alta incidência como é o caso de CaP. E ainda, preocupação em estudos sobre qualidade de vida (QV) e câncer em mulheres, porém, grande descaso em relação aos homens ${ }^{(2)}$

Um dos maiores desafios no tocante à detecção precoce do CaP é a falta de conhecimentos sobre sua história natural. Estudos demonstram que o CaP é histologicamente evidenciado em 30\% das necropsias em homens com idade igual e superior a 50 anos, sugerindo um curso latente prolongado, ou seja, em um grande contingente de homens a doença jamais evoluirá(3).

Achados no exame clínico combinados com o resultado da dosagem sérica do PSA podem sugerir a existência da doença. Nos casos suspeitos, é indicada biópsia prostática guiada por ultrassonografia transretal. O relatório anatomopatológico fornece a graduação histológica do sistema de Gleason, cujo objetivo é informar sobre a provável taxa de crescimento do tumor e sua tendência à disseminação, além de ajudar na determinação do melhor tratamento para o paciente ${ }^{(4)}$.

Os pacientes com CaP devem ser tratados em função do estágio da doença e neste sentido, a prostatectomia radical (PR) é considerada padrão ouro para o tratamento do CaP nos casos de tumores restritos à próstata com excelentes índices de sobrevida ${ }^{(4-5)}$.

Todas as modalidades terapêuticas apresentam risco significativo e efeitos colaterais, alterando com impacto negativo a QV dos pacientes. No caso da PR as principais complicações relacionam-se com estenose uretral, incontinência urinária (IU) e disfunção erétil (DE). Estudos apontam esses dois últimos como os principais fatores comprometedores da QV após a $\mathrm{PR}^{(4)}$.

Alterações de comportamento podem influenciar de forma decisiva a QV, a evolução da doença e o prognóstico. É fundamental que o diagnóstico seja associado a um exame psicodiagnóstico para adequado tratamento, inclusive quanto à aceitação da doença e como lidar com os sentimentos que emergem neste momento $^{(4)}$.

A oncologia é apontada como a especialidade que, por excelência, se viu confrontada com a necessidade de avaliar as condições de vida dos pacientes que tinham sua sobrevida aumentada devido aos tratamentos realizados, já que, muitas vezes, na busca de acrescentar anos à vida, era deixada de lado a necessidade de acrescentar vida aos anos ${ }^{(6)}$.

A fim de otimizar recursos disponíveis para melhorar os diferentes aspectos da QV no sistema de saúde, a relação entre QV e pacientes com câncer tem sido estudada abrangendo diferentes temas, tratamentos para a doença, controle de problemas, de sintomas e comorbidades. Outro interesse está diretamente ligado às práticas assistenciais dos serviços de saúde que tem a QV como indicador nos julgamentos clínicos de doenças específicas, nas decisões de condutas terapêuticas das equipes de saúde, além da avaliação do impacto físico e psicossocial $^{(2)}$.

Diante das dificuldades emocionais destes pacientes, diferentes profissionais da saúde devem pesquisar sobre esse tema a fim de oferecer uma atenção integral ao paciente e à família. Para tanto, é imprescindível a atuação de equipes multi e interdisciplinar já que nenhuma ciência é capaz sozinha de abranger todo o conhecimento necessário sobre um tema tão complexo como é a QV e câncer. O aumento do tempo de sobrevivência que não vem acompanhado de um desfrute da vida acarretaria mais sofrimento do que benefícios para esses pacientes, o que justifica pesquisas que envolvam QV nesses pacientes ${ }^{(2)}$.

Frente aos efeitos do diagnóstico e do tratamento cirúrgico, esse estudo propõe caracterizar a população quanto ao perfil sociodemográfico, clínico-cirúrgico, e presença de complicações cirúrgicas (DE e IU); descrever a QV e comparar as medidas de QV segundo o 
grupo etário e tempo pós-operatório em pacientes com CaP submetidos à PR nos cinco primeiros anos do pósoperatório.

\section{PROCEDI MENTOS METODOLÓGI COS}

Trata-se de um estudo observacional, descritivo e correlacional, tipo corte transversal. A população foi constituída de 187 pacientes submetidos à $P R$ no período de setembro de 2006 a setembro de 2011 em um hospital especializado em oncologia.

Participaram do estudo 81 pacientes que atenderam aos seguintes critérios de inclusão: residir em Uberaba; possuir diagnóstico de CaP localizado ou localmente avançado; ter sido submetidos à PR retropúbica com ou sem linfadenectomia; apresentar condições cognitivas para compreender as questões e ter período mínimo de três meses de cirurgia. Os critérios de exclusão foram: ter diagnóstico psiquiátrico de esquizofrenia ou transtorno bipolar, demência ou rebaixamento de consciência e aqueles que utilizarem medicamentos com efeitos no sistema nervoso central; ter sido submetido à PR de resgate ou ter sido realizada terapia adjuvante (tratamento hormonal, braquiterapia, radioterapia e quimioterapia) e não possuir informação sobre o estádio clínico do tumor no prontuário.

Antes da coleta de dados foi realizado teste piloto, sem reposição. Por meio do arquivo médico do hospital, obteve-se a relação dos pacientes que se incluíam no período. Mediante aleatoriedade simples, foram sorteados cinco indivíduos. A coleta de dados foi iniciada após conclusão do teste. Os pesquisadores, por meio de contato telefônico, convidaram os pacientes a comparecerem ao serviço em dia e local combinado, para a aplicação dos instrumentos. Nessa ocasião foram explicados os objetivos e importância da pesquisa, o direito de não participação sem qualquer prejuízo ao tratamento, o anonimato, sigilo e privacidade.

A primeira parte da coleta de dados foi realizada em uma sala ambulatorial do hospital previamente reservada e fora do horário de funcionamento dos ambulatórios de rotina. Tal estratégia visou garantir privacidade e conseguir, em tempo hábil, o retorno de um maior número de pacientes. Os formulários foram aplicados por um profissional de saúde, masculino, responsável pelo acompanhamento clínico do paciente. As perguntas foram realizadas em encontro único e em situação de privacidade, após a leitura e assinatura do Termo de Consentimento Livre e Esclarecido (TCLE).

A segunda parte da coleta foi desenvolvida junto ao serviço de arquivo médico do hospital com consulta ao prontuário a fim de obter informações sobre a história clínica e cirúrgica dos pacientes.

As variáveis as sociodemográficas e clínico-cirúrgicas estudadas foram: data de nascimento (dia, mês e ano); cor da pele (branca, parda, negra, amarela ou outra); condição educacional ( 1 grau incompleto, ou completo, 2ㅇ grau incompleto ou completo, 3 grau incompleto ou completo e pós-graduação); estado civil (solteiro, casado/amasiado, separado/divorciado e viúvo); número de dependentes (de nenhum a cinco ou mais); vínculos de emprego (um, dois, aposentado ou desempregado) e renda mensal em salários mínimos (não se aplica, 1 salário, de 2 a 3 , de 4 a 5 , de 6 a 7 e de 8 ou mais salários); tabagismo (sim, não e ex-tabagista); presença de hipertensão arterial, diabetes mellitus e doenças cardíacas; data da cirurgia; estadiamento cirúrgico (de T1a a T3c), valor do PSA pré-operatório $(\mathrm{ng} / \mathrm{ml})$, escore de Gleason total, peso $(\mathrm{kg})$ e altura $(\mathrm{m})$. A QV foi mensurada por meio dos domínios do European Organization for Research and Treatment of Cancer Quality of Life Questionnaire "Core" 30 Items (EORTCQLQ-C30)(7) , a função erétil pelo International Index of Erectile Function $\left(\right.$ IIEF-5) ${ }^{(8)}$ e a função urinária pelo International Consultation on Incontinence Questionnaire - Short Form (ICIQ-SF) ${ }^{(9)}$.

Concebido pela European Organization for Research and Treatment of Cancer (EORTC), o instrumento EORTC-QLQ-30 versão 3.0 em português, foi criado pela EORTC em 1986, validado e adaptado para a língua portuguesa. Inclui cinco escalas funcionais (função física, cognitiva, emocional, social e desempenho de papéis); três escalas de sintomas (fadiga, dor, náuseas e vômitos); uma escala de qualidade de vida e saúde global; seis outros itens que avaliam sintomas comumente relatados por doentes com câncer (dispnéia, falta de apetite, insônia, constipação e diarréia) e uma escala de impacto financeiro do tratamento e da doença. Os escores das escalas e das medidas dos itens individuais variam de zero a 100, sendo que um valor alto do escore representa um alto nível de resposta. Ou seja, um alto escore para a escala funcional representa um índice funcional saudável, enquanto que um escore alto para a escala de sintomas/itens representa um alto índice de sintomatologia/problema.

O IIEF-5 é um questionário com 15 itens, validado e traduzido para o português como uma escala autoaplicável para avaliar a função sexual. É válido do ponto de vista psicométrico e de fácil aplicação em pesquisas de ambiente clínico. Cinco fatores são identificados: função erétil, função orgásmica, desejo sexual, 
satisfação na relação sexual e satisfação global. De acordo com as respostas, a disfunção erétil pode ser classificada de grave a ausente. Considerando os objetivos desse estudo, foi avaliada a função erétil (questões de 1 a 5) e questão 15. Cada questão apresentada é seguida de escores numéricos, os quais apenas uma alternativa deve ser escolhida, que a seguir são transformados em escala de zero a 30 , classificandose conforme abaixo:

O ICIQ-S, traduzido para o português, apresenta propriedades psicométricas como validade, confiabilidade e responsividade para ambos os sexos. O ICIQ-SF é composto por seis questões, sendo o escore final ( 0 a 21) a soma dos escores das questões três, quatro e cinco. Nesse estudo foram descartadas as duas primeiras questões, permanecendo as três questões que compõem o escore, e a última, de caráter descritivo. Foram considerados incontinentes todos os homens que tiveram soma maior do que três.

Foi construída uma planilha eletrônica para armazenamento dos dados por meio do programa Microsoft ${ }^{\circledR}$ Office Excel ${ }^{\circledR}$ 2007. Os dados coletados foram digitados em dupla entrada para posterior verificação da consistência e consolidação. Os dados armazenados e validados no programa Microsoft ${ }^{\circledR}$ Office Excel ${ }^{\circledR} 2007$ foram importados para o aplicativo Statistical Package for Social Sciences (SPSS) versão 16.0.

Para os objetivos 1 e 2 foram utilizadas medidas descritivas, ou seja, foi realizada a distribuição de frequências para as variáveis categóricas e medidas de centralidade (média e mediana) e de dispersão (desvio padrão, valores mínimo e máximo) para as variáveis numéricas.

Para o objetivo 1 foi realizada categorização da idade em faixas etárias $(<40,40 \vdash 50,50 \vdash 60,60 \vdash 70$, $70 \vdash 80$ e $\geq 80$ ) e das variáveis cirúrgicas estádio (T1 a T2a, T2b e T2 a T3), escore de Gleason ( $\leq 6$, igual a 7 e $\geq 8)$ e PSA ( $\leq 4,4,1+10,10 \vdash 20$ e $\geq 20$ ) tornando-as categóricas ordinais. O tempo pós-operatório (variável quantitativa), obtido a partir da data de cirurgia, foi dicotomizado (até um ano de cirurgia e mais de um ano).

Para o objetivo 3 houve recategorização dos escores de DE e IU, tornando-as dicotômicas (presença e ausência) e da idade em dois grupos etários (adulto e idoso). Para a comparação da QV entre os grupos foi aplicado o teste não paramétrico Mann-Whitney, de acordo com os resultados do teste de normalidade de Shapiro Wilk. O nível de significância adotado foi $a=0,05$.
A consistência interna dos instrumentos (EORTC QLQ-C30, IIEF-5 e ICIQ) foi verificada através do Coeficiente de alfa de Cronbach, onde cada item de uma escala é testado simultaneamente com os outros. Os valores para o coeficiente de confiabilidade variam de 0 a 1 , isto é, quanto mais próximo de 1 é o coeficiente, mais consistente é o instrumento, sendo 0,7 considerado um valor de confiabilidade adequado.

O projeto foi encaminhado ao Comitê de Ética em Pesquisa com Seres Humanos da UFTM e, após sua aprovação pelo parecer consubstanciado sob protocolo número 2.099/2011, iniciou-se o teste piloto e a coleta dos dados. Os aspectos éticos foram baseados na Resolução 466/2012 sobre pesquisa envolvendo seres humanos.

\section{RESULTADOS}

Com relação às características sociodemográficas, a idade variou de 37 a 81 anos, sendo a média de 65,5 anos (D.P. $=8,4$ ), com $77,8 \%$ acima de 60 anos. Houve uma maior porcentagem de homens brancos (63\%) em relação aos negros (12,3\%). Apresentaram frequência maior os pacientes amasiados/casados $(80,3 \%)$ seguidos pelos divorciados/separados $(11,1 \%)$, semelhante relação de outro estudo. Foi observado que 54,3\% tinham de uma a duas pessoas como dependentes. A escolaridade com primeiro grau incompleto (84\%) e a renda mensal de um salário (54,3\%) foram os que apresentaram maior porcentagem. Quanto ao vínculo empregatício, a maioria era aposentada (75,3\%), o que não excluía na população outra fonte de renda, geralmente o exercício de atividades autônomas.

A análise da frequência das variáveis clínicas evidenciou uma maior proporção de homens extabagistas $(49,4 \%)$. Em relação às comorbidades, 53,1\% referiram HAS; $17,3 \%$ DM e $27,2 \%$ algum tipo de cardiopatia. Entretanto, 51,9\% deles estavam com peso saudável e $28,4 \%$ com sobrepeso.

Os pacientes foram questionados sobre o motivo que os levaram a procurar o serviço de urologia. A maioria procurou avaliação prostática de rotina $(85,2 \%)$, enquanto apenas $14,8 \%$ apresentavam sintomas urinários.

A análise da distribuição de fatores prognósticos utilizados atualmente (estádio cirúrgico, PSA préoperatório e escore de Gleason), a classificação de risco proposta por D'Amico e o tempo transcorrido entre a entrevista e a realização da cirurgia revelou que a maioria obteve escore de Gleason $\leq 6(63 \%)$ seguido daqueles com escore igual a $7(32,1 \%)$. O estadiamento 
cirúrgico mais frequente foi de T2c a T3 (74,1\%). A média do PSA foi de $8,4 \mathrm{ng} / \mathrm{ml}$, com variação de 1,2 a 27,8. O intervalo mais frequente foi o de $4,1+9,9$ $\mathrm{ng} / \mathrm{ml}$, conhecida como "zona cinza" de diagnóstico $(59,3 \%)$, seguida pelo intervalo de 10 - $20 \mathrm{ng} / \mathrm{ml}$ $(25,9 \%)$. Houve uma maior proporção no grupo de alto risco $(76,5 \%)$, seguido pelo grupo de baixo risco (14,8\%) considerando a classificação de D'Amico. O tempo médio de pós-operatório foi de 25,9 meses com variação de seis a 48,4 meses $( \pm 1,3)$, sendo que a maioria tinha realizado a PR há mais de um ano $(76,5 \%)$.

Em relação à $D E$, o IIEF-5 apresentou média de 6,9 $( \pm 10,2)$ nas respostas, com variação de escore de 1 a 30 pontos. O alfa de Cronbach de 0,99 indicou elevada consistência interna e homogeneidade dos itens. Foi observado que, após a cirurgia, a maioria dos pacientes não teve atividade sexual $(74,1 \%)$, não tentou penetração $(75,3 \%)$ e ainda apresentou muito baixa confiança em relação à ereção $(69,1 \%)$. De acordo com o escore do IIEF-5, 90,1\% dos pacientes apresentaram algum grau de $D E$, sendo DE grave em $75,3 \%$.

Em relação à IU, o ICIQ-SF apresentou um alfa de Cronbach de 0,90 indicando elevada consistência interna e homogeneidade dos itens. Observou-se frequência de $33,3 \%$ de incontinência, com média de escore de 3,26 $( \pm 5,37)$ e variação de 0 a 21 .

Quanto à QV, a Tabela 1 mostra uma análise descritiva simples por meio de mínimo, máximo, média, mediana, desvio padrão e o resultado do teste alfa de Cronbach para cada domínio da escala com mais de um item.

Tabela 1: Estatística descritiva das escalas do instrumento de qualidade de vida EORTC QLQ-C30 dos pacientes submetidos à prostatectomia radical. Uberaba, MG, Brasil, 2012.

\begin{tabular}{|c|c|c|c|c|c|c|}
\hline & Mínimo & Máximo & Média & Mediana & DP & $\mathbf{A}$ \\
\hline \multicolumn{7}{|l|}{ Escalas Funcionais } \\
\hline Função física & 33,3 & 100,0 & 96,5 & 100,0 & 10,1 & 0,78 \\
\hline Desempenho de papéis & 0 & 100,0 & 97,7 & 100,0 & 12,3 & 0,89 \\
\hline Função emocional & 0 & 100,0 & 80,7 & 100,0 & 26,2 & 0,90 \\
\hline Função cognitiva & 50 & 100,0 & 96,3 & 100,0 & 9,5 & 0,53 \\
\hline Função social & 66,7 & 100,0 & 97,9 & 100,0 & 6,7 & 0,22 \\
\hline \multicolumn{7}{|l|}{ Escalas de Sintomas } \\
\hline Fadiga & 0 & 33,3 & 6,4 & 0 & 10,5 & 0,33 \\
\hline Náuseas e vômitos & 0 & 33,3 & 0,4 & 0 & 3,7 & 1,0 \\
\hline Dor & 0 & 100,0 & 6,2 & 0 & 15,0 & 0,68 \\
\hline \multicolumn{7}{|l|}{ Sintomas } \\
\hline Dispneia & 0 & 100,0 & 8,6 & 0 & 21,6 & - \\
\hline Insônia & 0 & 100,0 & 20,6 & 0 & 30,5 & - \\
\hline Falta de apetite & 0 & 100,00 & 8,2 & 0 & 25,0 & - \\
\hline Constipação & 0 & 100,0 & 4,9 & 0 & 15,0 & - \\
\hline Diarreia & 0 & 33,3 & 0,82 & 0 & 5,2 & - \\
\hline Dificuldade Financeira & 0 & 100,0 & 16,5 & 0 & 29,4 & - \\
\hline Estado Global de Saúde & 8,33 & 100,0 & 69,7 & 66,6 & 18,3 & 0,82 \\
\hline
\end{tabular}

Para as escalas funcionais, os resultados das médias foram superiores a 80 pontos, revelando pouco comprometimento da QV. Dentre eles, o menor escore foi em relação à função emocional $(80,7)$ e o maior, desempenho de papéis $(97,7)$. Para as escalas de sintomas, os escores foram baixos evidenciando pouca influência na QV. A fadiga apresentou o maior escore $(6,4)$ e o item náuseas e vômitos, o menor $(0,4)$. Em relação aos sintomas (itens), a insônia teve maior pontuação $(20,6)$ e a diarreia a menor $(4,9)$. A dificuldade financeira teve um escore de 16,5 e a EGS um escore alto de 69,7.

No questionário EORTC QLC-C30, do total de nove escalas, cinco apresentaram coeficiente alfa de Cronbach acima de 0,70: função física, desempenho de papéis, função emocional, náusea e vômito, e EGS.
QV entre os adultos e idosos são apresentados a seguir na Tabela 2. 
Tabela 2: Comparação dos escores de qualidade de vida segundo o grupo etário dos pacientes submetidos à prostatectomia radical. Uberaba, MG, Brasil, 2012.

\begin{tabular}{|c|c|c|c|c|c|c|c|}
\hline \multirow{3}{*}{ Itens da Escala } & \multicolumn{6}{|c|}{ Grupo etário } & \multirow[b]{3}{*}{ p* } \\
\hline & \multicolumn{3}{|c|}{ Adulto } & \multicolumn{3}{|c|}{ I doso } & \\
\hline & Média & Mediana & Posto Médio & Média & Mediana & Posto Médio & \\
\hline Função física & 95,9 & 100 & 41,9 & 97,2 & 100 & 40,9 & 0,75 \\
\hline Desempenho de papéis & 98,7 & 100 & 40,7 & 98,7 & 100 & 41,1 & 0,86 \\
\hline Função emocional & 63,9 & 62,5 & 30,9 & 85,9 & 100 & 43,9 & 0,03 \\
\hline Função cognitiva & 93,5 & 100 & 38,2 & 97,1 & 100 & 41,8 & 0,36 \\
\hline Função social & 97,2 & 100 & 40,4 & 98,2 & 100 & 41,2 & 0,81 \\
\hline Fadiga & 9,9 & 5,6 & 47,9 & 5,5 & 0 & 38,0 & 0,09 \\
\hline Náuseas e vômitos & - & - & 40,5 & 0,5 & 0 & 41,1 & 0,60 \\
\hline Dor & 13,9 & 0 & 47,2 & 4,0 & 0 & 39,2 & 0,09 \\
\hline Dispneia & 9,3 & 0 & 40,8 & 8,5 & 0 & 41,1 & 0,95 \\
\hline Insônia & 24,1 & 0 & 42,2 & 19,6 & 0 & 40,7 & 0,78 \\
\hline Falta de apetite & 14,8 & 0 & 45,0 & 6,4 & 0 & 39,9 & 0,15 \\
\hline Constipação & 3,7 & 0 & 40,4 & 5,3 & 0 & 41,2 & 0,84 \\
\hline Diarreia & 1,9 & 0 & 42,3 & 0,5 & 0 & 40,7 & 0,34 \\
\hline Dificuldade financeira & 33,3 & 0 & 49,4 & 11,6 & 0 & 38,6 & 0,03 \\
\hline EGS & 61,6 & 66,7 & 30,0 & 72,0 & 75,0 & 44,1 & 0,02 \\
\hline
\end{tabular}

Os resultados aqui apresentados mostram que os itens desempenho de papéis, função emocional, função cognitiva, função social, estado global de saúde, náuseas e vômitos, dispneia, e constipação, tiveram seus postos médios aumentados, tendo apresentado diferença significativa apenas a função emocional $(p=0,03)$ e o EGS $(p=0,02)$. Esses aumentos indicam melhor $Q V$ nos cinco primeiros itens e pior QV nos três últimos itens na faixa etária acima de 60 anos. Com relação aos itens função física, fadiga, dor, insônia, falta de apetite, diarreia e dificuldade financeira pode ser verificado que os postos médios dos idosos foram menores, significando pior QV nesse grupo, apenas no item função física $(p=0,03)$.

A Tabela 3 indica os dados referentes à QV entre os paciente submetidos à $P R$ há menos de um ano e aqueles submetidos ao procedimento há mais de um ano.

Tabela 3: Comparação dos escores de qualidade de vida segundo o tempo pós-operatório dos pacientes submetidos à prostatectomia radical. Uberaba, MG, Brasil, 2012.

\begin{tabular}{|c|c|c|c|c|c|c|c|}
\hline \multirow{3}{*}{ I tens da Escala } & \multicolumn{6}{|c|}{ Tempo pós-operatório } & \multirow{3}{*}{ p* } \\
\hline & \multicolumn{3}{|c|}{ Até um ano } & \multicolumn{3}{|c|}{ Mais de um ano } & \\
\hline & Média & Mediana & Posto Médio & Média & Mediana & Posto Médio & \\
\hline Função física & 95,8 & 100 & 42,0 & 97,3 & 100 & 40,7 & 0,72 \\
\hline Desempenho de papéis & 94,7 & 100 & 40,8 & 98,7 & 100 & 41,1 & 0,91 \\
\hline Função emocional & 66,7 & 83,3 & 32,8 & 85,3 & 100 & 43,5 & 0,06 \\
\hline Função cognitiva & 91,2 & 100 & 34,3 & 97,8 & 100 & 43,1 & 0,03 \\
\hline Função social & 96,5 & 100 & 38,56 & 98,4 & 100 & 41,8 & 0,32 \\
\hline Fadiga & 11,1 & 11,1 & 49,4 & 5,0 & 0 & 38,4 & 0,03 \\
\hline Náuseas e vômitos & - & - & 40,5 & 0,5 & 0 & 41,2 & 0,58 \\
\hline Dor & 12,3 & 0 & 42,8 & 4,3 & 0 & 40,5 & 0,62 \\
\hline Dispneia & 12,3 & 0 & 42,8 & 7,5 & 0 & 40,5 & 0,57 \\
\hline Insônia & 26,3 & 0 & 45,2 & 18,8 & 0 & 39,7 & 0,31 \\
\hline Falta de apetite & 14,0 & 0 & 44,5 & 6,5 & 0 & 39,9 & 0,19 \\
\hline Constipação & 3,5 & 0 & 40,2 & 5,4 & 0 & 41,2 & 0,77 \\
\hline Diarreia & 1,8 & 0 & 42,1 & 0,5 & 0 & 40,7 & 0,37 \\
\hline Dificuldade financeira & 31,6 & 0 & 49,6 & 11,8 & 0 & 38,4 & 0,21 \\
\hline EGS & 65,0 & 66,7 & 65,7 & 71,1 & 66,7 & 42,6 & 0,25 \\
\hline
\end{tabular}

Os resultados mostram que os itens desempenho de papéis, função emocional, função cognitiva, função social, náuseas e vômitos e constipação tiveram seus postos médios maiores nos pacientes com mais tempo de cirurgia, tendo apresentado diferença significativa apenas a função cognitiva $(p=0,03)$. Assim, constata-se uma melhor QV nos quatro primeiros itens e pior QV nos últimos dois itens nos pacientes que possuem mais de um ano de cirurgia. Com relação aos itens fadiga, dor, dispneia, insônia, falta de apetite, diarreia, dificuldade financeira, função física e EGS foi verificado que os postos médios nos pacientes com mais tempo de cirurgia 
foram menores, sendo significativa apenas a fadiga $(p=0,03)$. Assim, verificou-se uma pior $Q V$ naqueles pacientes com mais tempo de cirurgia apenas nos dois últimos itens.

\section{SCUSSÃO}

O CaP é considerado um câncer da terceira idade uma vez que três quartos dos casos no mundo ocorrem a partir dos 65 anos. A incidência do CaP aumenta com a idade, atingindo quase $50 \%$ dos indivíduos com 80 anos, e assinala que esse tumor, provavelmente, não poupará nenhum homem que viver até $100 \operatorname{anos}^{(10)}$. Segundo o INCA, o único fator de risco bem estabelecido para o desenvolvimento do $\mathrm{CaP}$ é a idade e, aproximadamente $62 \%$ dos casos diagnosticados no mundo acometem homens com 65 anos ou mais. Com o aumento da expectativa de vida mundial, é esperado que o número de casos novos de câncer de próstata aumente cerca de $60 \%$ até o ano de $2015^{(1)}$. No presente estudo, a média etária foi de 65,7 anos. Resultados semelhantes foram encontrados em outros estudos. Em uma coorte hospitalar composta por pacientes com CaP localizado, a média foi de 66 anos ( 50 - 87), sendo 63 anos a média para aqueles que foram submetidos à $\mathrm{PR}^{(11)}$. Outro estudo a partir de espécimes cirúrgicos apresentou uma média de 64 anos $(47+85)^{(12)}$. Outro, mostrou um valor de 66 anos ( $43+77$ ) com $81 \%$ acima de 60 anos $^{(4)}$.

Embora a literatura apresente que o CaP é mais comum em negros do que em brancos, neste estudo houve uma maior porcentagem de homens brancos, o que pode estar associado ao fato de não ter sido avaliada a história familiar dos pacientes, que somada à idade e à raça/etnia, também é considerada importante fator de risco para esse tipo de neoplasia ${ }^{(1)}$.

Baixa escolaridade e baixa renda encontrada podem ser um indicativo de que esses pacientes vivem em condições precárias, decorrentes da baixa escolaridade e reduzida qualificação ocupacional, tendo acesso limitado aos serviços públicos, como educação e saúde. Esses resultados podem estar relacionados ao local do estudo.

A maioria era aposentada com exercício de atividades autônomas. Esse resultado foi encontrado em outro estudo em que mais da metade da amostra apresentava atividade profissional mesmo quando os indivíduos eram aposentados ${ }^{(4)}$.

O tabagismo é considerado pela OMS a principal causa de morte evitável em todo o mundo e estima-se que um terço da população mundial adulta seja fumante. Pesquisas comprovam que aproximadamente $47 \%$ da população masculina no mundo fumam ${ }^{(13)}$. Esse dado corrobora com a elevada porcentagem de homens que fumaram em algum período da vida nesse estudo.

Existe relação direta e linear da pressão arterial com a idade, sendo a prevalência de HAS superior a $60 \%$ na população acima de 65 anos. Assim, o elevado índice de hipertensos nesse estudo pode estar relacionado com a frequência de idosos e ainda com o hábito tabagista, fator de risco para a HAS ${ }^{(14)}$.

Em relação à etiologia do $\mathrm{CaP}$, são ressaltados os fatores de risco relacionados à idade e hereditariedade, contudo, alguns fatores exógenos como a alimentação, fatores virais e inflamatórios, podem ter importante papel no desenvolvimento do $\mathrm{CaP}$ e impacto no risco de progressão do câncer latente para a sua forma clínica(15). Esses fatores, geralmente se associam com condições clínicas tais como HAS, DM e cardiopatias que podem ainda funcionar como potenciais complicadores no pósoperatório. Assim, os resultados das variáveis clínicas desse estudo são dados importantes que possivelmente se associam com a etiologia ou com as complicações cirúrgicas (DE e IU).

A maioria procurou avaliação prostática de rotina sem qualquer sintomatologia urinária. Uma das peculiaridades do CaP é justamente a capacidade de ser encontrada em um número elevado de indivíduos sem causar qualquer mal. Um estudo da próstata por meio de necropsias de homens com idade entre 61 e 70 anos que faleceram sem doença prostática aparente, revelou focos neoplásicos em $24 \%$ deles. Contudo, apenas $11 \%$ dos indivíduos dessa faixa etária apresentam, em vida, manifestações clínicas relacionadas com o câncer. Assim, $13 \%$ dos tumores nesse grupo etário têm caráter indolente, são assintomáticos e seus portadores morrem por outros motivos, com o câncer, mas não pelo câncer ${ }^{(16)}$. As manifestações clínicas geralmente ocorrem, quando a neoplasia atinge a cápsula prostática, ou seja, quando a doença já se apresenta relativamente avançada. As manifestações mais comuns nesses casos são urinárias: disfunção miccional e hematúria( ${ }^{(4)}$.

Muitos tumores clinicamente classificados como localizados não o são de fato, levando à indicações terapêuticas curativas não efetivas. Por outro lado, pacientes com CaP sem significância clínica são tratados desnecessariamente em função da limitação atual da classificação prognóstica pré-tratamento do CaP localizado. Essa imprecisão torna-se um grave problema de saúde pública e justifica os estudos em desenvolvimento sobre novos fatores para nortear a melhor opção terapêutica para cada paciente ${ }^{(11)}$.

Considerando as características cirúrgicas, o escore 
de Gleason $\leq 6$ foi o mais frequente seguido daqueles com escore igual a sete. A maioria teve estadiamento cirúrgico de T2c a T3. Outros estudos mostraram proporções semelhantes tanto para o Gleason quanto para o estadiamento ${ }^{(12,17)}$. Outros dois, apenas para o Gleason, sendo o estádio mais frequente o T1c $(60 \%$ e $77 \%)^{(18-19)}$. O escore de Gleason relaciona-se com a extensão da doença, particularmente com o risco de acometimento extraprostático, assumindo valor prognóstico independente. Considera-se que, tumores com escore igual ou maior que sete são biologicamente agressivos, com escore de cinco ou seis são tumores com agressividade intermediária e os situados entre dois e quatro, agressividade biologicamente menor. A graduação no sistema Gleason baseia-se na diferenciação glandular e no padrão de crescimento em relação ao estroma. Nesse sistema não são consideradas as atipias nucleares e, até hoje, não foi demonstrado superioridade da avaliação nuclear em relação ao padrão glandular no $\mathrm{CaP}^{(17)}$.

Apesar de importantes, tanto a classificação de Gleason quanto o PSA total não definem perfeitamente o prognóstico dos pacientes com CaP localizado. Foi observado que mesmo com uma pontuação $\leq 6$ na classificação de Gleason, apenas 70\% dos pacientes têm a confirmação de doença localizada no estadiamento patológico, enquanto que mesmo com uma pontuação $\geq 7$ este percentual diminui para $34 \%{ }^{(11)}$.

A média de PSA pré-operatório de $8,4 \mathrm{ng} / \mathrm{ml}$ foi semelhante a de um estudo longitudinal em pacientes submetidos à PR que apresentou resultado de 7,8 (2,3 $24,0)$ contemplando $70 \%$ dos pacientes na zona cinza ${ }^{(18)}$. Outros estudos também obtiveram maior percentual de PSA inferior à $10 \mathrm{ng} / \mathrm{ml}(2,5+10 \mathrm{ng} / \mathrm{ml})$, respectivamente $80 \%$ e $70 \%{ }^{(18-19)}$. O PSA é marcador tecidual, porém não tumor específico. É uma glicoproteína excretada pelos ductos prostáticos para luz uretral como parte do plasma seminal. Apresenta sensibilidade e especificidade que não permitem sua utilização como método isolado. Estima-se que a sensibilidade do PSA para detectar doença restrita ao órgão, com uma especificidade de $100 \%$, seja de apenas $14 \%$. Com sua aplicação na clínica, houve um aumento no diagnóstico de tumores bem ou moderadamente diferenciados, não palpáveis ao toque retal. O PSA permitiu ainda o diagnóstico mais precoce, um maior índice de cura, ou mesmo colocar os pacientes em vigilância clínica ${ }^{(11)}$.

Mesmo com as limitações que tangenciam a classificação do CaP localizado, vários estudos respaldam a utilidade clínica dos marcadores prognósticos disponíveis atualmente: valores de PSA pré-tratamento, estadiamento e grau de diferenciação histológica conforme classificação de Gleason ${ }^{(11)}$.

O CaP é uma doença heterogênea com diferentes características de comportamento, e por isso define subgrupos com alto risco de recorrência após tratamento local. Nesse sentido houve prevalência do grupo de alto risco de acordo com a classificação de D'Amico, que é uma tentativa de antecipar o comportamento do $\mathrm{CaP}^{(16)}$.

A decisão do uso do rastreamento como estratégia de saúde pública deve se basear em evidências científicas de qualidade. No momento, não existem evidências de que o rastreamento para o CaP identifique homens que precisem de tratamento ou de que esta prática reduza a mortalidade pela doença ${ }^{(3)}$. Apesar do grupo The United States Preventive Services Task Force (USPSTF) ter emitido parecer contra o rastreamento, frequentemente nota-se pacientes com $\mathrm{CaP}$ em estadiamento acima de $\mathrm{T} 2 \mathrm{~b}$ e totalmente assintomáticos; certamente tais pacientes perderiam a chance de receberem um tratamento curativo se não fosse o rastreamento. Essa afirmação corrobora com os achados do presente trabalho onde a maioria não apresentava quaisquer sintomas, tinha estádios de T2c a T3 e tumores de alto risco. Nesse contexto, a grande questão não se resumiria em fazer ou não o rastreamento e sim em encontrar meios de distinguir os tumores indolentes daqueles dignos de tratamento.

Em relação à $D E$, foi observado que, após a cirurgia, a maioria dos pacientes não teve atividade sexual, não tentou penetração, apresentou muito baixa confiança em relação à ereção, sendo que a maioria mostrou algum grau de DE. Esses dados coincidem com os da literatura, na qual as taxas de impotência relatadas são discrepantes e altas, variando entre 60 a $90 \%{ }^{(20)}$.

Nesse estudo a taxa de IU se mostrou coerente com a literatura, haja vista as variáveis que geram grande amplitude em seus índices. Vale ressaltar que não foi realizada a classificação da IU e foram considerados incontinentes os pacientes que apresentaram qualquer perda. Taxas de IU variam consideravelmente e isso pode estar relacionado às diferenças nas abordagens cirúrgicas, nas definições de IU, nas metodologias dos estudos bem como o tempo de seguimento e instrumentos de avaliação da função urinária. Existe na literatura variação de $8 \%$ a $77 \%{ }^{(21)}$. A IU moderada ou grave está presente em $3 \%$ a $5 \%$ dos casos quando a intervenção é realizada por equipes habilitadas ${ }^{(10)}$.

É importante elucidar que embora o objetivo principal de qualquer tratamento para CaP seja 
maximizar a esperança de vida, os pacientes e a equipe de saúde precisam estar atentos ao impacto das terapias atuais sobre os resultados na QV. De acordo com os dados apresentados, a QV em geral foi considerada boa. As altas médias do EGS e da QV são explicadas pelo fato da média de sintomas ser baixa e demais funções altas. Esse resultado pode estar relacionado à ausência de sintomatologia característica do CaP. Resultados similares ao presente estudo foram encontrados na literatura utilizando EORTC QLQ-C30(22). Outro trabalho transversal foi observada diminuição discreta da QV após um ano da PR, sendo significativos os itens: funções física, emocional e fadiga ${ }^{(23)}$. Com o Short-Form Health Survey (SF-36), um estudo nacional observou ausência de diferenças significativas nos domínios de QV antes e após a $\mathrm{PR}^{(4)}$. Aplicando o Prostate Cancer Outcomes Study (PCOS) em mais de 2.000 pacientes, foi observado que $69,1 \%$ referiram preservar as relações sociais ${ }^{(24)}$.

Em relação à QV e o grupo etário, resultado semelhante ao presente estudo foi encontrado na literatura, ou seja, escores menores de QV (SF-36) nos domínios relacionados aos Aspectos Emocionais e Sociais em pacientes com menos de $60 \operatorname{anos}^{(4)}$. Isso pode ser justificado por esse grupo apresentar maior preocupação com o desempenho sexual e sequelas decorrentes da cirurgia.

\section{CONCLUSÃO}

Nesse estudo observou-se que a maioria dos pacientes submetidos à prostatectomia radical (PR) são idosos e assintomáticos. Em relação ao CaP, prevaleceu o estadiamento de T2c a T3 $(74,1 \%)$, Gleason $\leq 6$ (63\%), PSA pré-operatório de 4,1 a $10 \mathrm{ng} / \mathrm{ml}(59,3 \%)$ e, de acordo com a classificação de D'Amico, risco alto (76,6\%). A disfunção erétil esteve presente em 90,1\% e a incontinência urinária em 33,3\%. A maioria tinha mais de um ano de cirurgia (76,5\%). A qualidade de vida (QV)

\section{REFERÊNCIAS}

1. Instituto Nacional de Câncer [Internet]. Rio de Janeiro. 2014 [cited 2014 mai 17]. Estimativa 2014: incidência de câncer no Brasil. Available from:

http://www.inca.gov.br/estimativa/2014/estimativa-

24042014.pdf

2. Bertan FC, Castro EK. Qualidade de vida e câncer: revisão sistemática de artigos brasileiros. Psico. 2009;40(3):3665-3672. 3. Instituto Nacional de Câncer [Internet]. Rio de Janeiro. 2011 [cited 2011 out 17]. Rastreamento para o câncer de próstata. Diretrizes. Available from:

http://www.inca.gov.br/inca/Arquivos/publicacoes/diretriz rastre amento prostata.pdf

4. Vieira, ACOA. O impacto da doença e tratamento cirúrgico em homens acometidos por câncer de próstata: estudo exploratório de qualidade de vida. [Dissertação]. [São Paulo]: Faculdade de apresentou altos índices nas escalas funcionais e na escala global de saúde, e baixos na sintomatologia. Verificou-se melhor QV nos idosos em relação aos adultos nos itens: função emocional, dificuldade financeira e saúde global, e naqueles com mais de um ano de cirurgia nos itens: função cognitiva e fadiga. Embora não tenham sido observados grandes impactos na QV, houve diferenças significativas entre os grupos etários e o tempo pós-operatório. Ao considerar a subjetividade e a complexidade da temática estudada, esse estudo sinaliza a necessidade de outras pesquisas, uma vez que a QV reflete condições e valores individuais, podendo ser modificadas de acordo com as características de um determinado momento da vida das pessoas. Sobre a cirurgia, a PR é apenas uma modalidade terapêutica, sendo necessárias investigações complementares sobre as outras a fim de comparar os impactos na QV e buscar o melhor tratamento.

Os cuidados ao paciente com CaP geralmente são decorrentes de um empenho multidisciplinar. A enfermagem exerce um papel fundamental, pois é o grupo profissional que mantém contato com o paciente e sua família, em todos os períodos da doença e em diferentes contextos de atendimento. Porém, para fornecer um cuidado com qualidade, é preciso que o se tenha conhecimento das dimensões biológicas, fisiológicas e psicológicas da doença, dos tratamentos e do impacto desses na vida dos pacientes e familiares.

Assim, percebe-se a necessidade de subsídios sobre a população masculina com CaP. É preciso mudanças no perfil assistencial através de treinamento e educação continuada, a fim de oferecer qualidade de informações, auxílio psicológico, formação de grupos de apoio e outras medidas, já que a sobrevida que não é acompanhada de QV pode não ser a melhor opção para o paciente, causando mais sofrimento.

Medicina/USP; 2010.113p.

5. Frota R, Turna B, Barros R, Gill IS. Comparison of radical prostatectomy techniques: open, laparoscopic and robotic assisted. Int Braz J Urol. 2008; 34(3): 259-269.

6. Fleck MPA, Leal OF, Louzada S, Xavier M, Chachamovich E, Vieira $G$, et al. Desenvolvimento da versão em português do instrumento de avaliação de qualidade de vida da OMS (WHOQOL-100). Rev Bras Psiquiatr. 1999;21(1)19-28. 7. European organization for research and treatment of cancer (EORTC). European Organization for Research and Treatment of Cancer - Quality of Life - Questionnaire "CORE" 30 Items(EORTC QLQ C30). Version 3.0. EORTC Study Group on Quality of Life, 3. ed. Brussels, 2001.

8. Pechorro PS, Calvinho AM, Pereira NM, Vieira RX. Validação de uma versão portuguesa do Índice Internacional de Função

Eréctil-5 (IIEF-5). Revista Internacional de Andrologia, 
Barcelona, v. 9, n. 1, p. 3-9, jan. 2011.

9. Tamanini JTN, Dambros M, D'Ancona AL, Palma PCR, Netto J $r$ NR. Validação para o português do "International Consultation on Incontinence Questionnaire - Short Form" (ICIQ-SF). Revista de Saúde Pública, São Paulo, v. 38, n. 3, p. 438-44, jun. 2004. 10. Srougi M, Ribeiro LA, Piovesan AC, Colombo JR, Nesrallah A. Doenças da próstata. Rev Med. 2008;87(3): 166-177.

11. Migowski A, Azevedo e Silva G. Sobrevida e fatores prognósticos de pacientes com câncer de próstata clinicamente localizado. Rev Saude Publica. 2010;44(2):344-352.

12. Cambruzzi, E, Zettler CG, Pegas KL, Teixeira SL. Relação entre escore de Gleason e fatores prognósticos no adenocarcinoma acinar de próstata. J Bras Patol Med Lab. 2010;46(1):61-68.

13. Instituto Nacional de Câncer [Internet]. Rio de Janeiro. 2014 [cited 2014 mai 17]. Tabagismo: Dados e números. Available from:

http://www.inca.gov.br/tabagismo/frameset.asp?item=dadosnu m\&link =mundo.htm

14. Souza WSB, Jardim TV, Carneiro SB, Jardim PCBV.

Epidemiologia e orientações para o tratamento da hipertensão

em idosos. Rev Bras Hipertens. 2012; 19(3):65-69.

15. Rhoden EL, Averbeck MA. Câncer de próstata localizado. Rev AMRIGS. 2010;54(1): 92-99.

16. Dall'oglio MF, Crippa A. Câncer de próstata localmente avançado. In: Nardozza JR, A, Zerati FIlho, M, Reis RB. Urologia fundamental. São Paulo: Planmark; 2010. p.217.

17. Nassif AE, Filho RT, Gomes de Paula RX, Taguchi WS, Pozzobon $\mathrm{HJ}$. Perfil epidemiológico e fatores prognósticos no tratamento cirúrgico do adenocarcinoma de próstata clinicamente localizado. Rev Col Bras Cir. 2009; 36(4):327-331.

18. Makarov DV, Trock BJ, Humphreys EB, Mangold LA, Walsh PC, Epstein JI, et al. Updated nomogram to predict pathologic stage of prostate cancer given prostate-specific antigen level, clinical stage, and biopsy Gleason score (Partin Tables) based on cases from 2000 to 2005. Urology. 2007;69(6): 1095-1101. 19. Partin AW, Mangold LA, Lamm DM, Walsh PC, Epstein JI, Pearson JD. Contemporary update of prostate cancer staging nomograms (Partin Tables) for the new millennium. Urology. 2001; 58(6): 843-848.

20. Thompson I, Thrasher JB, Aus G, Burnett AL, Canby-Hagino ED, Cookson MS, et al. Guidelines for the management of clinically localized prostate cancer: 2007 update. J Urol. 2007; 177(6): 2106-2131.

21. Klingler $C$, Marberger $M$. Incontinence after prostatectomy: surgical treatment options. Curr Opin Urol. 2006; 16(2):60-64. 22. Wyler SF, Ruszat R, Straumann U, Forster TH, Provenzano M, Sulser $\mathrm{T}$, et al. Short, intermediate, and long-term quality of life after laparoscopic radical prostatectomy: does the learning curve of LRP have a negative impact on patients' quality of life? Eur Urol. 2007; 51(4): 1004-1012.

23. Lilleby W, Fossa SD, Waehre HR, Olsen DR. Long-term morbidity and quality of life in patients with localized prostate cancer undergoing definitive radiotherapy or radical prostatectomy. Int J Radiat Oncol Biol Phys. 1999;43(4):735743.

24. Hoffman RM, Hunt WC, Gilliland FD, Stephenson RA, Potosky AL. Patient satisfaction with treatment decisions for clinically localized prostate carcinoma. Results from the Prostate Cancer Outcomes Study. Cancer. 2003; 97(7):1653-1662.

Artigo recebido em 30/11/2012.

Aprovado para publicação em 06/05/2014.

Artigo publicado em 30/09/2014. 